\title{
Automatic Implementation and Simulation of Qualitative Cognitive Maps"
}

\author{
João Paulo Carvalho and José Alberto Tomé \\ IST - Instituto Superior Técnico \\ INESC - Instituto de Engenharia de Sistemas e Computadores \\ R. Alves Redol, 9, 1000 LISBOA, PORTUGAL \\ joao.carvalho@inesc.pt jose.tome@inesc.pt
}

\begin{abstract}
This short paper presents the overview of an ongoing project which goal is to obtain and simulate the dynamics of qualitative systems through the combination of the properties of Fuzzy Boolean Networks and Fuzzy Rule Based Cognitive Maps.
\end{abstract}

\section{Introduction}

Decision makers, whether they are social scientists, politicians or economists, usually face serious difficulties when approaching significant, real-world dynamic systems. Such systems are composed of a number of dynamic concepts interrelated in complex ways, usually including feedback links that propagate influences in complicated chains. Axelrod work on Cognitive Maps (CMs) [1] introduced a way to represent real-world qualitative dynamic systems, and several methods and tools [2,3] have been developed to analyze the structure of CMs. However, complete, efficient and practical mechanisms to analyze and predict the evolution of data in CMs are necessary but not yet available for several reasons [4]. System Dynamics tools like those developed by J.W.Forrester [5] could be a solution, but since in CMs numerical data may be uncertain or hard to come by, and the formulation of a mathematical model may be difficult, costly or even impossible, then efforts to introduce knowledge on these systems should rely on natural language arguments in the absence of formal models. Fuzzy Cognitive Maps (FCM), as introduced by Kosko [6], are an alternative approach to system dynamics. However, in most applications, a FCM is indeed a man-trained Neural Network that doesn't share the qualitative properties of other fuzzy systems [7], and the modeled system ends up being represented by a quantitative matrix without any qualitative knowledge. Therefore, we can say that there are currently no tools available to adequately represent the dynamics of qualitative systems.

Even if we had the tools to represent and analyze those systems, a different issue would be the building of a qualitative CM. The standard methodology for acquisition of the necessary data relies usually on lengthy processes of individual and simplistic

1 This work is partially supported by the program POSI (under the EU 3rd framework program) and by the Portuguese Foundation for Science and Technology (FCT)

V.N. Alexandrov et al. (Eds.): ICCS 2001, LNCS 2074, pp. 217-220, 2001.

(C) Springer-Verlag Berlin Heidelberg 2001 
information collection: the result ends up being a list of quantitative relevant world concepts displayed as a matrix where weights represent the relations between those concepts $[2,3,4,6]$. Therefore we end up with quantitative CMs where any vestiges of a system with real-world rich qualitative entities and rich qualitative relations were simply ignored. Besides, there is the additional problem of combining the views of different analysts into one single map, which is usually done by simple math operations between the resulting matrixes.

This paper presents a general overview of an ongoing work that pretends to potentiate two original research lines to solve the problems presented above.

The first research line is in the potential use of really Qualitative Cognitive Maps as a tool not only to describe but also to simulate scenarios in qualitative real world systems like Social Conflicts, Political Science cases or Economic real-world problems. Fuzzy Rulebased Cognitive Maps (RB-FCM) $[7,8,9,10]$ were introduced in previous works and are still being developed. They provide new Fuzzy Operators and a complete methodology for analysis and design of the dynamics of qualitative systems.

The second research line develops a new class of Boolean Neural Nets - Fuzzy Boolean Networks (FBN) [11,12,13] - which behave like fuzzy systems and are capable of non supervised learning of rules and of using the learned rules in qualitative reasoning.

\section{Automatic Implementation and Simulation of Cognitive Maps}

This paper purposes and introduces the overall architecture of AISCMap (Automatic Implementation and Simulation of Cognitive Maps). AISCMap should automatically generate a RB-FCM using FBN for the acquisition of the rules, concepts and relations that compose the map, allowing the simulation of what-if scenarios in the qualitative system that it models. The following sections provide a simplified description of AISCMap.

\subsection{Inputs for AISCMAP}

The inputs for AISCMap consist of the output provided by expert's analysis of the system to be modeled. Even after debate, each expert might have its own view of the problem, and a preferred way of representing the system. They can use natural language to express concepts and the rules relating them; they can provide examples of possible relations and their effect; they can define what is the type of relation involving two or more concepts; they can describe the system using matrixes, etc.

\subsection{The Fuzzy Boolean Network Module}

The FBN module extracts RB-FCM concepts, rules and relations from the expert raw data combining them into a single qualitative cognitive map. The map is described using RB-FCMSyntax, which is a language developed to provide a complete description of a RB-FCM. 
Neural Networks are known for their capability in apprehending relationships among variables, but is also well known their difficulty in expliciting these relations into human comprehensive rules [15]. Fuzzy logic based systems, are very useful in explaining behaviors by a set of qualitative rules [14], but are not so good in which concerns learning of those rules. A synergetic cooperation between these two paradigms leads usually to hybrid systems that can learn and also explain the relationships by rules. However, these hybrid systems are usually an artificial engineered human work, where algorithms as well as fuzzy and neural components are "inserted" into the system. Although being based on common grounds, FBN are a new class of neural fuzzy systems that don't have many things in common with other neuro-fuzzy systems. Fuzzy qualitative reasoning is a natural and emergent property of Boolean networks[11,12]. These nets present many similarities with natural systems. They are capable of learning rules from non-supervised experiments in an Hebbian-like manner and they can automatically adapt to the granularity[13] of the antecedent variables and select the relevant ones. FBN are Boolean Nets with Macroscopic Emergent Fuzzy Reasoning, which allows them to extract qualitative rules from experimental data [11,12]. Here are some of the main FBN features:

- Variables/Concepts are associated with neural areas

- Values are given by neural activation

- Local Random Neural Connections

- Macro Structured (Links connecting Areas)

- Hebbian like Nonsupervised Learning by experiments

- Embedded Variable Selection and Granular adaptation

\subsection{From FBN to RB-FCM}

In AISCMap it is necessary to extend the working principles of the FBN to the problem of RB-FCM. This involves the adequate definition of the relationship between concepts and structure of the Maps and the neural areas and sub-areas as defined on the FBN.

\subsection{Representing and Modeling Real World Qualitative Dynamic Systems}

The RB-FCM allow a representation of complex real-world dynamic qualitative systems and the simulation of events and their influence in the system. RB-FCM are essentially composed of fuzzy Concepts and fuzzy Relations. Concepts are fuzzy variables that represent the value or the change in Actors, or Social, Political, Economical or Abstract entities that compose the system we are trying to model $[7,8,9,10]$.

Relations between concepts are defined using fuzzy rule bases and can be of several types: Fuzzy Causal; Fuzzy Influence; Possibilistic; Invariant and Time variant Probabilistic; Possibilistic and Probabilistic; Fuzzy Similarity; Level/Change [10].

RB-FCM are iterative and provide mechanisms to simulate the dynamics of the system it represents (including fuzzy mechanisms to simulate time, which is not a 
given issue when relations are not time dependent mathematical equations as in system dynamics tools). The evolution of the system through time might converge in a single state or a cycle of states under certain conditions [7].

One of the main important uses for a dynamic model of a system, is the analysis of "WHAT-IF" scenarios ("What happens to the system when some event occurs?"), since the change of state in one or more concepts or relations affect the system in ways that are usually difficult or impossible to predict due to complex feedback links. RB-FCM are well adapted to these analysis, since introduction or removal of concepts and/or relations are possible and easily done without the hassle of the exponential increase of rules usually associated with fuzzy rule based systems [7,9].

\section{Conclusions}

AISCMAP is an ongoing work that is the result of a synergic approach of two different areas. Most of its modules are on an advanced stage of evolution, and the obtained results are promising, but there is still a lot of work to be done especially on the interaction between the expert results and the inputs to the system; and on the interaction FBN/RB-FCM.

\section{References}

[1] Axelrod,R.,The Structure of Decision: Cognitive Maps of Political Elites, Princeton University Press, 1976

[2] Laukkanen,M.,Comparative Cause Mapping of Management Cognition:A computer Database For Natural Data,Helsinki School of Economics and Business Publications, 1992

[3] Decision Explorer, Banxia Software

[4] Laukkanen,M.Conducting Causal Mapping Research:Opportunities and Challenges, in Managerial and Organisational Cognition,edited by Eden, C.and Spender, Sage, 1998

[5] Forrester, J.W., several papers available in http://sysdyn.mit.edu/sd-intro/home.html

[6] Kosko,B., "Fuzzy Cognitive Maps", Int. Journal of Man-Machine Studies, 1986

[7] Carvalho,J.P.,Tomé, J.A.,"Rule Based Fuzzy Cognitive Maps and Fuzzy Cognitive Maps A Comparative Study", Proceedings of the 18th International Conference of the North American Fuzzy Information Processing Society, NAFIPS99, New York

[8] Carvalho, J.P. , Tomé, J.A.,"Rule Based Fuzzy Cognitive Maps- Fuzzy Causal Relations", Computational Intelligence for Modelling, Control and Automation, Edited by M. Mohammadian, 1999

[9] Carvalho,J.P.,Tomé,J.A., "Fuzzy Mechanisms For Causal Relations", Proceedings of the Eighth International Fuzzy Systems Association World Congress, IFSA'99, Taiwan

[10] Carvalho,J.P.,Tomé, J.A.,"Rule Based Fuzzy Cognitive Maps - Qualitative Systems Dynamics", Proceedings of the 19th International Conference of the North American Fuzzy Information Processing Society, NAFIPS2000, Atlanta

[11] Tomé, J.A., "Counting Boolean Networks are Universal Approximators", Proceedings of the 1998 Conference of the North American Fuzzy Information Processing Society, Florida

[12] Tomé,J.A., "Neural Activation ratio based Fuzzy Reasoning", Proceedings of the 1998 IEEE World Congress on Computational Inteligence, Anchorage, Alaska

[13] Tomé, J.A., "Automatic Variable Selection and Granular Adaptation in Fuzzy Boolean Nets", Proceedings of the 18th International Conference of the North American Fuzzy Information Processing Society, NAFIPS99, New York

[14] Mamdani,E.and Gaines,B.,Fuzzy "Reasoning and its Applications".London: Academic Press, 1981.

[15] Rumelhart,D.,G.Hinton,R.Williams "Learning Internal representations by error propagation" Parallel Distributed Processing,volI,chap.8.Cambridge,MA,MIT Press, 1986 